\title{
The Teaching Development and Performance Art of Trumpet
}

\author{
Wang Lei \\ Xi'an Conservatory of Music, Room 20320 East Building Zuolin Youre Community, \#136 Jianxi \\ Road Peilin District Xi'an, Shaanxi Province,Tel: 18609284444
}

Keywords: trumpet; teaching development; performance art

\begin{abstract}
As one of the most ancient brass instruments in the west, trumpet is the ideal instrument for solo, ensemble, concert and concerto due to its wide range, excellent voice and strong penetrability. Since it was brought in into China, it develops rapidly and Chinese trumpets works and performance are deeply engraved with national features. In current Chinese musical field, wind trumpet is popular among people and it plays an important role in the symphony orchestra; it is especially true for it is of great significance for the performance of symphony orchestra. This paper will study the teaching development and performance art.
\end{abstract}

\section{Introduction}

Among the various instruments, trumpet is widely loved by people due to its rich musical performance and loud musical features, thus playing an important role in the current music development. As performers of trumpets, he should equip with excellent trumpet performance art and should make due contributions for cultivating more trumpet performers. Therefore, this paper would analyze from the performance art and teaching development of trumpet.

\section{Best rhythm techniques of trumpets}

The performance of trumpets lies in the grasp of performance techniques for trumpets. In other words, by better grasping of performance techniques, performers can perform loud and beautiful music. In order to have a good grasp of the performance techniques, performers should better coordinate and harmonize the breathing organs and mouth muscles so as to maintain the flexibility and powerful state of mouth. As for breathing, no matter how people breathe, the throats should not be hindered. During breathing, however, it should maximum the absorbed air at the same time of maintaining the relax state so as to better expand the thorax and abdominal muscle. As for expiration, it should expire despite the hindering of mouth so as to maintain the evenly, stable and fluent feature of airflow. During the performance of trumpet, expiration is the first link during the creation of music while the phrases would affect the expiration quality and quantity. As performers of trumpet, only by expiration and inspiration based on the quality, quantity and voice during the performance, can it realize the excellent music performance under more comfortable and nature state.

\section{Performance art of trumpets}

\section{Extension of trumpet range}

During the symphony orchestra performance, it is not only necessary to accurately express the note of the composition but also requires the proper extension of range. By obeying the composition of trumpet, performers should continue and harmonize $\mathrm{C}$ note so as to perform charming note. During the soprano performance, it often ensures the demands of gas during performance process for note by increasing the mouth muscle strengthens and increasing original respiratory volume. For trumpet performance, due to the changes of age and physical quality, performers requires long time training to constantly increase the respiratory volume. As for the performance of high notes, it should extend the low note district. At present, according to the performance methods of modern trumpets, the needed sound effect does not require to play the low notes. By training in the 
above-mentioned methods, it can satisfy the performance demands and obtain the more proper range.

\section{The tonal changes of art}

During the performance of symphony orchestra, tonal change is a more complex technique. As for the ordinary trumpet performance, it should clearly distinguish the transpositional methods, especially the zone with more tones. Only by clearly distinguishing the tonal changes, it can powerfully guarantee the best effects of performance. Most works clearly distinguish the parts of trumpets while tonal changes are one of the mostly-applied performance method. By changes between inD and inE, it can confer trumpet performance with stronger presentation with changes of tones. For example, in a performance of capriccio, during the initial stage of performance, trumpets can perform the strong artistic features of horns by strong expressive effect and with thick tonal atmosphere. In the tones presented by this method, it can notate by inE method while some parts requires notating with inF method. During this process, it only requires to clarify and determine the western performance technique so as to make the performance style more comprehensive and artistic value more abundant.

\section{Trumpet performance techniques}

Trumpet has the features of wide application range and appreciated by refined and popular tastes, thus being fond of people, especially the youth. Many young people actively participate in the learning of trumpet performance. During trumpet performance, breathing method is the basic foundation for performance as well as the basic force for phonation. Therefore, it should effectively control the intonation, strength, quality and voice during performing trumpets. In real performance, there are three breathing methods, among which one is thoracic breathing that requires the extraction of the muscles of ribs and sternum so as to maximization of the air volume of expiration and inspiration and the exhale according to the demands of performance. Inhale and exhale is finished in the expansion and shrinking of chest. The second is belly respiration which contracts and expands the muscles of diaphragm movement and belly muscles so as to finish the exhale and inhale. In other words, the expansion and contraction of gas is controlled by diaphragm movement and belly muscles. When people inhale, the diaphragm movement can increase the chest and belly outline and external air would come into the belly through lung. During exhaling, it can exhale the air by the lung pressure due to the contraction of belly muscles and it can come into the trumpet pipe. The third is thoracic-abdominal respiration that combines the chest breath under the basis of belly respiration.

During trumpet performance, performers are not born with breathing techniques; instead it is obtained by training. As for respiratory methods, combined breathing method is one important training methods during the trumpet performance, which goes through the trumpet learning during the first day of learning trumpet and it is also one important learning part during performance.

\section{Playing postures}

During the trumpet performance, it requires the standing posture and sitting posture. No matter which one you choose, the correctness of posture should be ensured for it is the basic guarantee for the effective application of trumpet breathing. Correct sitting posture can protect the performer's breathing organs. On the other hand, it can ensure the smooth breath so as to lay good foundation for the normal performance and affect the development and application of different technology of trumpet performance. One leg on the other and too lower of head as well as the tight clamping of ribs by two arms, these are abnormal performance postures which would hinder the normal operation of breathing organs during performance, which can bring disadvantage and negative factors for performance and would finally affect the performance effect. Therefore, it should arouse our due attention during the actual performance.

\section{Correct breathing}

1) Inspiration

Before inspiration, it requires good playing preparation. In other words, put the trumpet mouth on your lips and inhale quickly with nose and mouth so as to contract lower your diaphragme and 
expand ribs towards the ribs. During inspiration process, it is finished with the help of rib and diaphragme outline, which is also the most reasonable and most effective breathing method. Meanwhile, inspiration in this method, it should pay attention to the relax state of breathing muscles so as to ensure the smooth and nature expiration of breath. As for respiratory volume, it should be decided based on the length of phrases so as to avoid the influence of unnecessary expiration on the stability of breath.

2) Expiration

After finishing inspiration, it should transfer the breath steadily and evenly into the trumpet mouth so as to make beautiful sound. During correct inspiration, it should ensure that the expired breath can form smooth, full, steady and even airflow which is closely related with the hindering of expiration. By the existence of hindering, it can ensure the steadiness of airflow. During performance of trumpet, it should control the airflow by the combined effect of control on organs below diaphragme, tongue tip, buccal fissure and throat.

\section{Teaching development of trumpets}

The learning of trumpet is closely related with long-term breath training. By the correct application of breath, performers can effectively overcome the difficulty during playing. During the trumpet teaching, it can strengthen the training of following two situations.

\section{Without playing trumpets}

This method is convenient and easy and it can help performers enhance the development movement of chest and increase the power of muscle tissue. For example, the diaphragme training is to train the strength of breath muscle. In practical training, it should slowly breathe by playing the palms on the belly and then repress the pressure of diaphragme muscles. When it is full of inspiration, it should then exhale the air evenly and steadily. In concrete training, it should avoid the maximization of inspiration and expiration and should control freely and use of the two links so as to avoid the nervousness of muscle training.

\section{By playing trumpets}

During trumpet learning, prolonged sound playing is an inseparable training content. For the prolonged sound is simple and pure, it would involve the basic training of many aspects and can fully taste the relations of mouth shape, tongues and breath of performers. By the training of this part, it can practice their own tissue techniques and apply the techniques into real performance.

\section{Conclusion}

Performance of trumpets is widely loved by people and trumpets are important in large symphony for it is widely applicable. In the previous content, the teaching development and performance art is studied to some extent, which requires mastering the key points in teaching practices of trumpets so as to obtain better performance effects.

\section{References}

[1] Wang Hongxing. Thinking on the professional trumpets teaching for music majors in higher normal colleges [J].Modern Communication.2012 (09).

[2] Shang Lixing. Exploration on the new teaching methods of trumpets in higher normal colleges [J].Art Education. 2010(10).

[3] Zhao Danning. Several Enlightenments in trumpets teaching [J].The World of Music. 2009(02).

[4] Song Ying. Several Thinking on cultivating best trumpets performance mentality [J]. Yellow River of the Song. 2015(12).

[5] Zhang Jialin. Analysis on the effect and status of trumpets in symphony orchestra [J].Music Time. 2015(20). 
[6] Niu Xueli. Analysis on trumpets in the folk music [J].Theatrical Family. 2015(19).

[7] Ma Honggang. Study on the training content and methods of breath training in trumpet performance [J].The World of Music. 2011(01). 